FORMATION Formation emploi

Revue française de sciences sociales

149 | Janvier-Mars 2020

Varia

\title{
Les jeunes NEET : résistances et évolutions sur vingt ans
}

NEET Youth : resistance and evolution over twenty years

Junge Schulabgänger ohne Abschluss : Widerstände und Entwicklungen in den letzten zwanzig Jahren

Jóvenes NEET : resistencias y evoluciones en veinte años

\section{Magali Danner, Christine Guégnard et Olivier Joseph}

\section{(2) OpenEdition}

Journals

Édition électronique

URL : https://journals.openedition.org/formationemploi/8059

DOI : 10.4000/formationemploi.8059

ISSN : 2107-0946

Éditeur

La Documentation française

Édition imprimée

Date de publication : 31 mars 2020

Pagination : 61-85

ISSN : 0759-6340

Référence électronique

Magali Danner, Christine Guégnard et Olivier Joseph, «Les jeunes NEET : résistances et évolutions sur vingt ans », Formation emploi [En ligne], 149 | Janvier-Mars 2020, mis en ligne le 01 janvier 2022, consulté le 06 janvier 2022. URL : http://journals.openedition.org/formationemploi/8059 ; DOI : https://doi.org/10.4000/formationemploi.8059

(c) Tous droits réservés 


\title{
Les jeunes NEET : résistances et évolutions sur vingt ans
}

\author{
Magali Danner \\ Enseignante chercheure en sciences de l'éducation, Institut de recherche sur l'éducation \\ (IREDU, EA 7318), Université Bourgogne Franche-Comté
}

ChRISTINE GUÉGNARD Chargée d'études en sciences de l'éducation, Institut de recherche sur l'éducation (IREDU, EA 7318), Centre associé au Céreq, Université Bourgogne Franche-Comté

OLIVIER JOSEPH Économiste, chargé d'études au Département des entrées et évolutions dans la vie active,

Céreq

Résumé

Les jeunes NEET : résistances et évolutions sur vingt ans

En France, près de deux millions de jeunes ne sont ni en étude, ni en emploi, ni en formation. Cet article présente les traits dominants concernant ces NEET, les résistances ou les évolutions identifiables sur une durée de vingt ans, à travers quatre enquêtes menées par le Céreq. Si les situations des femmes et des hommes semblent se rapprocher sur le marché du travail, les jeunes femmes n'en sortent pas gagnantes pour autant au regard de leurs trajectoires dans des contextes marqués par des transformations tant structurelles que conjoncturelles. Au prisme d'une analyse genrée, l'article revient sur l'importance de dépasser l'utilité statistique du concept NEET, en raison de sa dimension statique.

Mots clés : jeune, origine sociale, non diplômé, insertion professionnelle, femme, division sexuelle du travail, chômage des jeunes, condition de vie

Abstract

\section{NEET Youth: resistance and evolution over twenty years}

In France, nearly two million young people are neither in education, employment nor training. This article presents the dominant profiles concerning these NEETs, the resistances or identifiable evolutions over a period of twenty years, through four surveys conducted by the Céreq. While the situations of women and men seem to be getting closer in the labour market, young women do not win in terms of their trajectories in contexts marked by both structural and economic transformations. Through the prism of a gender analysis, the article discusses the importance of overcoming the statistical utility of the NEET concept, because of its static dimension. 
Keywords: young person, social origin, non graduate, transition from school to work, woman, gender based division of labour, youth unemployment, living condition

Journal of Economic Literature: J 24, I 39

Traduction : auteur.e.s

\section{Introduction}

Depuis plus de trois décennies, l'insertion professionnelle des jeunes constitue une préoccupation majeure dans la société française, notamment en contexte de récession économique où ils sont surexposés au risque de non-emploi. Pour appréhender cette réalité, la Commission européenne a introduit un nouvel indicateur en 2010 : celui de NEET, contraction de l'expression anglaise Not in Education, Employment or Training ${ }^{1}$. Il permet ainsi de mesurer la part des jeunes déscolarisés, sans emploi et ne suivant aucune formation, parmi l'ensemble de la population de la même catégorie d'âge. En France, l'OCDE (Organisation de coopération et de développement économiques) chiffre le nombre de NEET à près de deux millions de jeunes, représentant près de $17 \%$ des 15 à 29 ans pour l'année 2017 (précisément $18 \%$ des jeunes femmes et $15 \%$ des jeunes hommes).

Cet indicateur NEET a été présenté dans le cadre de la stratégie de croissance «Europe $2020 »$ comme une valeur supplémentaire (Eurofound, 2012) pour analyser la situation de la jeunesse en raison du manque de pertinence des indicateurs traditionnels du marché du travail. En effet, depuis la fin du XIX ${ }^{\mathrm{e}}$ siècle, moment de l'invention du chômage (Salais \& al. 1986), la catégorisation des personnes sans emploi et spécifiquement la mesure du chômage soulèvent des enjeux politiques, sociaux (Maruani, 2002 ; Freyssinet, 2009 ; Gautié, 2009) et des problèmes techniques statistiques. Des jeunes sans emploi peuvent ne pas être comptabilisés comme chômeurs car ils ne remplissent pas toutes les conditions ${ }^{2}$. Par exemple, certains souhaitent travailler, mais ne sont pas disponibles immédiatement ou n'ont pas fait de recherche active d'emploi, ou sont découragés et comptés comme inactifs ${ }^{3}$. L'indicateur NEET va donc au-delà des critères classiques et éclaire une réalité sociale juvénile en marge de l'emploi et de la formation.

Cependant, le terme NEET n'est pas tout à fait nouveau. Il est apparu au Royaume-Uni dans les années 90, dans un contexte social et politique particulier sous les qualificatifs de Status ZerO et Status A (Istance \& al., 1994 ; Williamson, 1997). Il ciblait les jeunes

1. Cette recherche sur les NEET fait partie d'un projet financé par l'Agence nationale de recherche (ANR15-ORAR-0005-01).

2. Selon la définition du Bureau international du travail (BIT), un chômeur est une personne en âge de travailler (15 ans ou plus) qui répond simultanément à trois conditions : être sans emploi, être disponible pour prendre un emploi dans les 15 jours, avoir cherché activement un emploi dans le mois précédent.

3. Cf. le halo du chômage retenu par l'Insee : personnes inactives, soit qui recherchent un emploi, mais ne sont pas disponibles, soit qui souhaitent travailler, mais ne recherchent pas d'emploi, qu'elles soient disponibles ou non (Bessone \& al., 2016). 
de 16-17 ans déscolarisés, en incapacité de percevoir des allocations chômage du fait du changement des régimes de protection sociale (Furlong, 2006). Il a été officiellement introduit suite à l'arrivée au pouvoir, en 1997, du Parti travailliste (New Labour), lors d'un rapport du gouvernement britannique afin de lutter contre l'exclusion des jeunes (Bridging the Gap: new opportunities for 16-18 year olds not in education, employment or training, Social Exclusion Unit, 1999).

Depuis, l'intérêt envers les NEET a augmenté, s'est étendu aux jeunes âgés de 15 à 24, puis aux 15-29 ans, pour devenir le cœur des lignes directrices des politiques européennes dans le cadre de la stratégie " Europe 2020 » (European Commission, 2010), des mesures pour l'emploi en 2012 (employment package cf. European Commission, 2012) et du programme de la Garantie jeunesse (European Council, 2013). Dans ce cadre européen, les jeunes NEET deviennent une catégorie cible de la politique de l'emploi pour la première fois, en France, via la Garantie jeunes, dès octobre $2013^{4}$.

Nombre d'études comparatives européennes soulignent une gamme de facteurs sociaux, économiques et personnels qui augmentent le risque de devenir NEET, certains jeunes cumulant les facteurs de vulnérabilité, comme un faible niveau d'éducation, un problème de santé ou de handicap, une origine immigrée, la parentalité, un milieu social et culturel défavorisé... Les jeunes femmes sont davantage susceptibles de se retrouver NEET, notamment après la naissance d'un enfant, en lien avec la répartition traditionnelle des rôles entre hommes et femmes. La dernière publication de la Fondation européenne pour l'amélioration des conditions de vie et de travail (Eurofound, 2016) souligne aussi la diversité de cette population à partir des enquêtes Forces de travail 5 . Elle différencie sept groupes distincts : chômeurs de courte ou de longue durée, malades et handicapés, chargés de famille, découragés, jeunes en attente d'un emploi ou d'une formation immédiate, autres inactifs. En 2013, la population NEET en France se distingue des pays européens par une part plus importante de jeunes au chômage depuis moins de douze mois (30\%, chiffre au-dessus de la moyenne européenne de $25 \%$ ).

Sept ans auparavant, l'OCDE (2009) avait étudié les NEET, en France, en construisant un pseudo-panel à partir de l'enquête Emploi ${ }^{6}$. Les résultats montraient des retours fréquents en NEET et le quart des jeunes avaient connu une expérience de NEET au cours des dix-huit mois suivant leur sortie de formation en 2003. Il s'agissait principalement de jeunes sans diplôme, d'origine immigrée et vivant dans des quartiers défavorisés. Ces résultats corroboraient une autre étude plus ancienne de l'OCDE. Ciblant plus spécifi-

4. En février 2013, le Conseil européen a lancé l'Initiative pour l'emploi des jeunes, avec un fonds de plus de six milliards d'euros et la Garantie jeunes a été expérimentée, dès octobre 2013, dans quelques territoires français, puis généralisée à l'ensemble de la France en 2017. Ce dispositif récent, mis en œuvre par les Missions locales, permet d'accompagner les jeunes entre 16 et 25 ans, en situation de grande précarité, vers l'emploi ou la formation avec une allocation financière (Escudero, Mourelo, 2018).

5. Enquêtes par sondage auprès des ménages.

6. En ciblant les sortants de formation en 2003 durant 18 mois et trois ans avant 2003. 
quement les 15-24 ans en France, la part de NEET apparaissait parmi les plus élevées, avec l'Espagne et la Grèce, sur une période d'observation de cinq ans, de 1998 à 2003 (Quintini, Martin, 2006). Néanmoins, les allers-retours vers une situation de NEET étaient plus importants en France.

Si ces travaux permettent de mieux connaître la population, ils restent toutefois assez silencieux sur les dynamiques d'une telle expérience jusqu'à la dernière recherche faite par Carcillo \& al. (2015) qui démontrent que les jeunes décrocheurs constituent une part substantielle des NEET et ont tendance à le rester ${ }^{7}$. Seulement quelques études déterminent les raisons de devenir NEET, mais aucune n'identifie de quelle manière le passage en NEET affecte la suite des trajectoires (Ryan, 2001). Les parcours et modes de vie de ces jeunes n'ont pas encore fait l'objet d'une enquête spécifique en France, cette population reste à découvrir au-delà du portrait statistique. Tel est notre objectif ici à travers deux questions qui s'entrecroisent. En premier lieu, les jeunes restés aux portes de l'entreprise et de l'école présentent-ils des caractéristiques spécifiques qui ont évolué sur vingt ans ? Cette optique temporelle se justifie par les difficultés persistantes d'accès à l'emploi des jeunes sur un marché du travail en fluctuation au gré de la conjoncture économique observée durant ces années. En second lieu, les expériences de NEET se déclinent-elles de la même manière au féminin et au masculin au fil du temps ? Ce second aspect renvoie à l'analyse des disparités des comportements d'offre de travail selon le genre. Certes, l'inégalité des jeunes hommes et des jeunes femmes face au chômage s'est réduite depuis le milieu des années 1990 (Coquet, 2004) ; cependant, plus on s'éloigne du chômage conventionnel, plus on rencontre de femmes (Maruani, 2004 ; GuergoatLarivière, Lemière, 2014). Sans emploi, de nombreuses femmes " invisibles " considérées comme inactives, au foyer auparavant, se trouvent assimilées aux NEET. Nous cherchons ainsi à mieux comprendre leur situation en dépassant les indicateurs habituels et ce, dans un contexte d'augmentation du taux d'activité féminine, d'un rapprochement des conditions d'accès à l'emploi entre jeunes femmes et jeunes hommes au cours des années (Couppié, Épiphane, 2018).

Afin de répondre à ces interrogations, notre recherche s'appuiera sur les données du Centre d'études et de recherches sur les qualifications (Céreq) qui retracent les cinq premières années suivant la fin des études de quatre cohortes sorties de tous niveaux de formation initiale (en 1992, 1998, 2004 et 2010) (cf. encadré 1). Ces enquêtes Génération ${ }^{8}$ fournissent une richesse d'informations pour analyser la transition de l'école à l'emploi,

7. En utilisant trois panels (European Union Statistics on Income and Living Conditions (EU-SILC), statistiques de l'union européenne sur le revenu et les conditions de vie, 2008-2010), faisant ainsi figure de parcours longitudinal, 2434 jeunes de 16 à 20 ans ont été suivis durant 48 mois dans douze pays européens. Cependant, la plupart ne sont pas exclus du marché du travail, puisque $70 \%$ des jeunes poursuivent leurs études.

8. Ces jeunes ont en commun d'être sortis du système éducatif la même année, quel que soit le niveau ou le domaine de formation atteint, d'où le terme de "Génération " parfois dans la suite de l'article. 
en France, sur une période de vingt ans. Le recul de cinq ans, pour chaque enquête, renforce la qualité de l'analyse et la continuité (ou la disparition) dans le temps des disparités au moment de la transition professionnelle. En effet, il faut attendre plusieurs années pour que la stabilisation professionnelle soit établie pour le plus grand nombre de jeunes, l'insertion étant définie comme un processus complexe "socialement structuré ayant une certaine durée » (Rose, 2018 p. 17). De plus, les retraits du marché du travail peuvent être liés à des événements, notamment la parentalité durant les premières années de vie active.

\section{Encadré 1. Les données : un dispositif original et unique en France}

Les enquêtes Génération du Céreq permettent, depuis plus de vingt ans, à partir d'un panel représentatif de l'ensemble des jeunes quittant le système éducatif, une description précise de leur insertion professionnelle dans les cinq années suivant la fin des études. Des non-diplômés aux doctorants, tous les niveaux sont concernés, dans toutes les spécialités de formations suivies par voie scolaire ou en apprentissage. Les jeunes reconstituent, par enquête téléphonique, mois par mois leur situation (emploi, chômage, formation, inactivité), fournissent des informations sur les métiers exercés (contrat, temps de travail, statut, salaire, entreprise...), expriment leurs opinions quant à leur situation actuelle, perspectives professionnelles, motif d'arrêt des études... Les bases de données comparables des quatre enquêtes totalisent 69025 répondants (effectifs non pondérés). Ces quatre populations enquêtées sont constituées de jeunes qui ont pu être retrouvés et contactés via leurs établissements de formation. On ne peut pas exclure qu'une partie des NEET (Not in Education, Employment or Training ou ni en étude, ni en emploi, ni en formation), notamment ceux dont les modes de vie ne facilitent pas un suivi longitudinal, soient absents ou aient moins participé à ces enquêtes.

La première partie de l'article présentera les profils des jeunes, plutôt bien identifiés par la littérature scientifique, qui ne sont ni en emploi ni en formation. Si la part de NEET cinq ans après la fin des études s'est maintenue autour de $18 \%{ }^{9}$ pour les quatre enquêtes, ce chiffre global masque toutefois des disparités individuelles persistantes, mais aussi des changements. Le contexte éducatif et économique auquel chaque génération a été confrontée invite à s'intéresser à la modification de la composition de ce groupe de jeunes. De plus, dans le cadre d'un marché du travail français segmenté (Piore, 1978 ; Caroli, Gautié, 2009), la prise en compte des contrats de travail et des expériences de NEET permettra de savoir si les emplois de courte durée ou aidés sont des trappes à NEET.

Parmi les sortants suivis par le Céreq, vivre un temps de NEET ${ }^{10}$ est devenu un point de passage pour plus de $70 \%$ des jeunes au cours des cinq ans suivant la fin de leurs études. La deuxième partie mettra l'accent sur le poids des caractéristiques individuelles quant

9. La part de jeunes ni en étude, ni en emploi, ni en formation est calculée ici sur l'ensemble des sortants du système éducatif la même année (et non par rapport à l'ensemble de la catégorie d'âge comme dans les études internationales). NEET regroupe ainsi les situations de chômage et d'inactivité déclarées par les jeunes.

10. D’une durée d'un mois au moins et hors période de vacances. 
à la probabilité d'échapper à la situation de NEET lors de la première séquence. Ainsi, cette recherche longitudinale sur plusieurs cohortes vise à approfondir la connaissance sur les déterminants du passage (partie 1) et du maintien en situation de NEET (partie 2) durant une période de vingt ans, soulignant les spécificités qui façonnent les parcours des jeunes femmes sortant du système éducatif. Cette approche genrée permettra, en effet, de relever que cet indicateur commun qui facilite les comparaisons ne peut à lui seul résumer les problématiques à l'égard de l'insertion des jeunes. Le passage d'une analyse factuelle, fondée sur des résultats statistiques, à une analyse critique du concept NEET ouvre, dans une dernière partie, la réflexion sur les limites de cet indicateur et invite dès lors à porter un autre regard sur cette jeunesse.

\section{Portrait des jeunes NEET à l'épreuve du temps}

Il est une évidence sociologique de dire que les ressources sociales, économiques et culturelles apportées par le milieu familial peuvent interférer sur les parcours des jeunes (Bourdieu, Passeron, 1970 ; Coleman, 1988 ; Portes, 2000 ; Kramartz, Viarengo, 2015) et influencer la qualité de leur réseau social (Granovetter, 1995 ; de Larquier, Rieuquau, 2012).

Si les NEET à cinq ans se retrouvent dans tous les milieux sociaux, ils sont moins nombreux dans les familles dites "favorisées ». Ainsi en moyenne, seulement $12 \%$ de ces jeunes ont un père cadre supérieur (20\% pour la population en emploi et formation). En parallèle, près du tiers ont un père ouvrier (versus le quart des jeunes en emploi et formation). Quant aux mères des jeunes NEET, elles sont plus souvent au foyer : proche de $40 \%$ pour les deux premières cohortes et $30 \%$ pour les deux suivantes (versus $35 \%$ à $17 \%$ pour la population en emploi et formation). Parmi ces mères au foyer, la moitié d'entre elles n'ont jamais travaillé. Finalement, à la fin de leurs études, seulement $40 \%$ des jeunes avaient leurs deux parents en activité (pour $60 \%$ des jeunes en emploi et formation), soulignant un isolement parental du marché du travail. Par ailleurs, ces jeunes sont plus fréquemment issus d'une origine migratoire ${ }^{11}$, celle-ci peut constituer un obstacle à leur intégration professionnelle (Brinbaum, Guégnard, 2012 ; Guégnard \& al., 2015 ; Cusset \& al., 2015 ; France Stratégie, 2017).

Cependant, les ressources familiales ne sauraient expliquer à elles seules les trajectoires des jeunes sortants. Sur le plan structurel, le niveau d'études s'est élevé pendant ces vingt années, en lien avec les transformations des cursus scolaires, et notamment les réformes

11. En moyenne, $30 \%$ des jeunes ont leurs parents nés à l'étranger (versus $20 \%$ pour les jeunes non NEET). Parmi les parents d'origine étrangère, 8 à $12 \%$ viennent du Maghreb (versus 4 à $5 \%$ des non-NEET selon les cohortes). 
de l'enseignement professionnel. Le nombre important de jeunes non diplômé.e.s qui composent la population NEET (Tableau 1) ne doit pas occulter la proportion non négligeable, et relativement constante sur vingt ans, de titulaires de baccalauréats $(21 \%$ de bachelières et $15 \%$ de bacheliers en moyenne) et de diplômé.e.s de l'enseignement supérieur (18\% des femmes et $13 \%$ des hommes). La population NEET reste donc composite au fil des années, mais il convient de souligner que cette tendance à l'élévation des qualifications est davantage marquée pour la population féminine.

Ainsi, 30 \% des sortantes étaient diplômées de l'enseignement supérieur en 1992 (pour $28 \%$ des hommes), écart qui s'est creusé en vingt ans (40\% des femmes et $34 \%$ des hommes). Dès la Génération 1998, le nombre de titulaires de CAP-BEP ${ }^{12}$ a baissé, au profit des bachelières et des diplômées de l'enseignement supérieur. Toutefois, cette progression ne s'est guère accompagnée d'un bouleversement majeur dans leurs voies de prédilection, ni d'un positionnement différencié dans les filières de formation ${ }^{13}$. En écho, une sensible impulsion vers les diplômes supérieurs est aussi relevée du côté des hommes, avec un fléchissement des diplômés de CAP-BEP à partir de 2004. Néanmoins, la part des jeunes non diplômés, qui a diminué essentiellement entre les deux premières enquêtes suite à la suppression du palier d'orientation en fin de $5^{\mathrm{e}}$ en 1994 et donc des orientations vers les lycées professionnels (Defresne, Krop, 2016), demeure importante (14\% des sortantes et $23 \%$ des sortants en 2010). Or, le diplôme est un facteur déterminant de l'insertion professionnelle (Rose, 2005 ; Barret \& al., 2014). Il protège aussi du chômage (Martinelli, Minni, 2013) et facilite le retour à l'emploi. De ce point de vue, les femmes paraissent donc mieux armées pour se positionner sur le marché du travail : les deux tiers d'entre elles sont diplômées, pour la moitié seulement des hommes.

Tableau 1. Profil des jeunes NEET cinq ans après la fin des études (en \%)

\begin{tabular}{|lcc|cc|cc|cc|}
\hline & \multicolumn{2}{c|}{ Génération 92 } & \multicolumn{2}{c|}{ Génération 98 } & \multicolumn{2}{c|}{ Génération 2004 } & \multicolumn{2}{c|}{ Génération 2010 } \\
& Femmes & Hommes & Femmes & Hommes & Femmes & Hommes & Femmes & Hommes \\
\hline Non diplômé & 38 & 52 & 31 & 44 & 31 & 47 & 36 & 52 \\
CAP-BEP & 27 & 25 & 28 & 25 & 28 & 25 & 21 & 19 \\
Baccalauréat & 19 & 11 & 21 & 14 & 22 & 15 & 23 & 17 \\
Supérieur & 16 & 12 & 20 & 17 & 19 & 13 & 20 & 12 \\
Ensemble & 100 & 100 & 100 & 100 & 100 & 100 & 100 & 100 \\
\hline
\end{tabular}

Lecture: pour la Génération 2010, parmi les femmes NEET (ni en étude, ni en emploi, ni en formation), cinq ans après la fin de la scolarité, $36 \%$ sont non diplômées, $21 \%$ sont titulaires d'un CAP ou BEP (respectivement certificat d'aptitude professionnelle et brevet d'études professionnelles), $20 \%$ ont un diplôme du supérieur.

Source : Céreq, enquêtes comparables Génération 1992, 1998, 2004, 2010, interrogations à 5 ans.

12. Certificat d'aptitude professionnelle et Brevet d'études professionnelles.

13. Les femmes sont surreprésentées dans les spécialités tertiaires (ou services), tandis que les hommes dominent dans les spécialités industrielles (production). Aujourd'hui encore, filles et garçons ne suivent pas les mêmes filières scolaires, n'accèdent pas aux mêmes savoirs et cette formation différenciée les conduit vers des espaces professionnels distincts (Couppié, Épiphane, 2018). 
L'arrivée d'un enfant peut rapidement les amener à interrompre leur activité professionnelle. Toutes générations confondues, près du tiers des femmes avec enfant sont en situation d'inactivité ou de chômage, alors que le nombre de NEET parmi les pères demeure relativement bas (9\%). Ceci témoigne des rôles sociaux différenciés, notamment dans les responsabilités familiales : "l'inactivité est un statut qui demeure socialement admissible pour les femmes, difficilement pensable pour les hommes " (Maruani, 2004 , p. 106). Une analyse par cohorte souligne toutefois que la part de NEET parmi les femmes avec enfant a chuté (de 40 à $29 \%$ ) entre les Générations 1992 et 1998 et s'est maintenue autour du tiers par la suite. Le recul de l'âge de la maternité et de la mise en couple, depuis les années 1960 (Couppié, Épiphane, 2007 ; Daguet, Niel, 2010), va de pair avec l'augmentation de la durée de scolarisation, mais aussi avec la place croissante des femmes sur le marché du travail. La volonté de vivre un certain temps à deux avant de fonder une famille entre certainement aussi en jeu (Buisson, Daguet, 2012). Ces changements ont davantage affecté la population féminine, puisque le tiers des sortantes de formation initiale - pour les deux premières Générations - et le quart - pour les deux suivantes - ont eu un ou plusieurs enfants sur les cinq années d'observation ${ }^{14}$.

Ainsi, la conjugaison de ces facteurs (un niveau de formation plus élevé, un maintien plus marqué dans l'emploi et une propension moins forte à devenir mère) explique la forte diminution de l'écart conséquent observé lors de la première enquête entre la part de NEET à cinq ans chez les femmes $(25 \%)$ et chez les hommes (12\%) (Tableau 2). Dès la deuxième Génération, les femmes sont, en effet, nettement moins souvent dans cette situation (proche de $19 \%$ ), comparativement aux hommes dont la part de NEET progresse à partir de la cohorte 2004 (17\%), puis à $2 \%$ pour celle de 2010 .

14. Parmi les sortants, le nombre de pères passe de $15 \%$ pour les deux premières générations à $10 \%$ ensuite. 
Tableau 2. Situation des jeunes cinq ans après la fin de la formation (en \%)

\begin{tabular}{|lcc|cc|cc|cc|}
\hline & \multicolumn{2}{c|}{ Génération 92 } & \multicolumn{2}{c|}{ Génération $\mathbf{9 8}$} & \multicolumn{2}{c|}{ Génération 2004 } & \multicolumn{2}{c|}{ Génération 2010} \\
& Femmes & Hommes & Femmes & Hommes & Femmes & Hommes & Femmes & Hommes \\
\hline Emploi & 71 & 84 & 78 & 87 & 76 & 79 & 74 & 75 \\
NEET & 25 & 12 & 18 & 11 & 19 & 17 & 20 & 20 \\
Formation & 4 & 4 & 4 & 2 & 5 & 4 & 6 & 5 \\
\hline
\end{tabular}

Lecture : parmi les sortantes de la Génération 2010, 74 \% des femmes ont un emploi, $20 \%$ sont NEET (ni en étude, ni en emploi, ni en formation) et $6 \%$ en étude ou formation cinq ans après la fin des études.

Source : Céreq, enquêtes comparables Génération 1992, 1998, 2004, 2010, interrogations à 5 ans.

Une modélisation spécifique ${ }^{15}$ a été réalisée afin d'étudier les déterminants sociaux et scolaires sur la probabilité d'être NEET à cinq ans, indépendamment du contexte générationnel (voir Tableau 3). Toutes choses égales par ailleurs, elle confirme que les femmes avec enfant encourent plus de risques par rapport à une femme sans enfant d'être ni en emploi ni en formation. Les diplômes jouent un rôle positif et hiérarchisé auquel s'ajoute l'effet des spécialités (avec davantage de débouchés pour les formations industrielles) : les sortants sans diplôme sont les plus vulnérables face à la probabilité de devenir NEET, viennent ensuite les diplômés CAP-BEP, puis les bacheliers, ensuite les diplômés du supérieur. Par ailleurs, les jeunes quittant l'école en 2004 et en 2010 ont été pénalisés par la conjoncture économique (Joseph \& al., 2008 ; Barret \& al., op. cit.), les débutants étant surexposés aux aléas conjoncturels (Fondeur, Minni, 2004) qui réduisent leurs perspectives d'embauche.

15. L'ambition de notre analyse empirique est de mettre en évidence des effets de genre et des effets de caractéristiques sociales sur la probabilité d'être NEET à cinq ans. Pour étudier l'effet net de chaque variable et hiérarchiser leur poids sur la probabilité de se trouver en situation de NEET cinq ans après la fin des études, il est nécessaire de mobiliser des modélisations estimant les probabilités. Un modèle a été effectué selon la méthode d'Heckman en deux étapes : 1) sur la probabilité d'être NEET à cinq ans, 2) sur la probabilité d'être en situation de chômage versus inactivité tenant compte ainsi du biais de sélection (ou de l'occurrence d'être dans telle situation). Cette méthode permet des estimateurs non biaisés des paramètres associés à ces caractéristiques sur la probabilité d'être au chômage plutôt qu'en inactivité par la prise en compte de la sélection, en première étape, de la probabilité d'être NEET. Nous avons préféré présenter un modèle logistique polytomique distinguant chômage et inactivité plutôt qu'un modèle dichotomique (NEET vs non-NEET). Il s'agit de mettre en lumière des différences persistantes entre les femmes et les hommes, les premières étant plus souvent en inactivité (définie par la réponse de la personne qui affirme être sans emploi et ne pas en rechercher). Au cours des enquêtes du Céreq, la part des femmes en inactivité n'évolue pas (de 7 à $5 \%$ des sortantes pour 1 à $2 \%$ des hommes) et les femmes avec enfant sont aussi nombreuses à se déclarer au chômage ou en inactivité pour les trois dernières cohortes. 
Tableau 3. Probabilité d'être NEET cinq ans après la sortie de l'école (modèle d'Heckman)

\begin{tabular}{|c|c|c|c|}
\hline \multirow[t]{2}{*}{ Être au chômage versus en inactivité à 5 ans } & \multicolumn{3}{|c|}{ Coefficient } \\
\hline & Ensemble & Femmes & Hommes \\
\hline Constante & $1.563^{*}$ & $1.556^{*}$ & $1.70^{*}$ \\
\hline Enfant & & $-1.175^{*}$ & -0.119 \\
\hline Femme avec enfant Femme sans enfant (Réf.) & $-0.932^{*}$ & & \\
\hline Homme avec enfant & -0.018 & & \\
\hline Homme sans enfant & 0.089 & & \\
\hline Priorité la vie hors travail à 5 ans & $-0.536^{*}$ & $-0.587^{*}$ & $-0.456^{*}$ \\
\hline Situation convient à 5 ans & $-1.278^{*}$ & $-1.310^{*}$ & $-1.099^{*}$ \\
\hline Sans diplôme Sortie avec CAP-BEP (Réf.) & -0.035 & -0.021 & -0.121 \\
\hline Baccalauréat & -0.137 & 0.012 & $-0.423^{*}$ \\
\hline Diplôme Bac + 2 ou 3 & $-0.197^{*}$ & -0.129 & $-0.293^{*}$ \\
\hline Diplôme $\mathrm{Bac}+4$ ou plus & -0.051 & 0.072 & $-0.295^{*}$ \\
\hline Génération 1998 Génération 1992 (Réf.) & -0.089 & -0.018 & $-0.290^{*}$ \\
\hline Génération 2004 & $-0.272^{*}$ & $-0.260^{*}$ & $-0.345^{*}$ \\
\hline Génération 2010 & $-0.317^{*}$ & $-0.299^{*}$ & $-0.388^{*}$ \\
\hline \multicolumn{4}{|l|}{ Être en situation de NEET à 5 ans } \\
\hline Constante & $0.404^{*}$ & $0.66^{*}$ & $0.275^{*}$ \\
\hline Homme (Réf.) & $0.260^{*}$ & & \\
\hline Être en couple & $0.119^{*}$ & $0.385^{*}$ & $-0.353^{*}$ \\
\hline Retard scolaire en $6^{e}$ & 0.043 & 0.029 & $0.075^{*}$ \\
\hline Sortir d'apprentissage & -0.010 & 0.017 & -0.024 \\
\hline Sans diplôme Sortie avec un CAP-BEP (Réf.) & $0.217^{*}$ & $0.154^{*}$ & $0.259^{*}$ \\
\hline Baccalauréat & $-0.239^{*}$ & $-0.224^{*}$ & $-0.261^{*}$ \\
\hline Diplôme Bac + 2 ou 3 & $-0.369^{*}$ & $-0.370^{*}$ & $-0.309^{*}$ \\
\hline Diplôme Bac +4 ou plus & $-0.365^{*}$ & $-0.313^{*}$ & $-0.365^{*}$ \\
\hline Spécialité générale Spécialité industrielle (Réf.) & -0.018 & -0.050 & 0.022 \\
\hline Spécialité tertiaire (services) & $0.078^{*}$ & 0.038 & 0.064 \\
\hline Arrêt des études pour raison financière & $0.047^{*}$ & 0.011 & $0.109^{*}$ \\
\hline Arrêt des études par lassitude & 0.011 & 0.009 & -0.002 \\
\hline Parents indépendants Parents ouvriers employés (Réf.) & 0.033 & 0.033 & 0.036 \\
\hline Parents cadres & 0.025 & -0.005 & 0.080 \\
\hline Parents techniciens & 0.028 & 0.056 & 0.001 \\
\hline Deux parents en emploi à la fin des études & $-0.051^{*}$ & $-0.077^{*}$ & -0.031 \\
\hline Mère au foyer à la fin des études & $0.080^{*}$ & $0.085^{*}$ & 0.055 \\
\hline Deux parents français Parents autres origines (Réf.) & -0.040 & -0.034 & -0.052 \\
\hline Deux parents originaires d'Europe du sud & -0.008 & -0.008 & 0.004 \\
\hline Deux parents originaires du Maghreb & 0.034 & $0.079^{*}$ & -0.005 \\
\hline Ile-de-France Fin des études dans d'autres régions (Réf.) & 0.001 & -0.049 & 0.070 \\
\hline Nord-Pas-de-Calais & 0.023 & $0.058^{*}$ & -0.025 \\
\hline Alsace & -0.002 & -0.004 & -0.018 \\
\hline
\end{tabular}




\begin{tabular}{|lccc|}
\hline Bretagne & -0.000 & 0.005 & -0.009 \\
Pays-de-Loire & -0.025 & -0.028 & -0.012 \\
Aquitaine & $-0.061^{*}$ & -0.046 & -0.072 \\
Midi-Pyrénées & 0.026 & 0.020 & 0.031 \\
Rhône-Alpes & -0.001 & 0.018 & -0.005 \\
PACA (Provence Alpes-Côte d'Azur) & 0.025 & 0.014 & 0.031 \\
Taux de chômage régional à 4 ans & -0.032 & -0.059 & 0.029 \\
Avoir été NEET durant les 6 premiers mois (Réf.) & & & \\
Non NEET durant les 6 premiers mois & $-0.181^{*}$ & $-0.169^{*}$ & $-0.154^{*}$ \\
NEET à 3 ans & $0.377^{*}$ & $0.412^{*}$ & $0.299^{*}$ \\
NEET durant les 6 premiers mois et à 3 ans & $0.373^{*}$ & $0.418^{*}$ & $0.244^{*}$ \\
Un emploi à durée indéterminée & $-0.673^{*}$ & $-0.700^{*}$ & $-0.708^{*}$ \\
Un emploi d'une durée d'au moins 12 mois & $-0.498^{*}$ & $-0.511^{*}$ & $-0.505^{*}$ \\
Un emploi aidé & $-0.079^{*}$ & $-0.118^{*}$ & -0.029 \\
Différents emplois précaires & -0.017 & -0.043 & 0.024 \\
Priorité la vie hors travail à 5 ans & $0.178^{*}$ & $0.259^{*}$ & -0.011 \\
Situation à 5 ans convient & $-1.426^{*}$ & $-1.245^{*}$ & $-1.694^{*}$ \\
Génération 1998 & 0.007 & $-0.138^{*}$ & $0.225^{*}$ \\
Génération 2004 & $0.187^{*}$ & $-0.130^{*}$ & $0.507^{*}$ \\
Génération 2010 & $0.102^{*}$ & $-0.102^{*}$ & $0.379^{*}$ \\
\hline Log de vraissemblance & -25112.013 & -13726.212 & -10884.674 \\
athrho 1992 (Réf.) & $0.2166^{*}$ & $0.154^{*}$ & $0.235^{*}$ \\
\hline
\end{tabular}

Sont indiqués les résultats significatifs aux seuils de $5 \%$.

Lecture : toutes choses égales par ailleurs, une femme a plus de risques d'être NEET cinq ans après sa sortie de formation par rapport à un homme (coefficient positif de 0.260 significatif au seuil de $5 \%$ ).

Sigles : CAP-BEP : respectivement certificat d'aptitude professionnelle et brevet d'études professionnelles.

Source : Céreq, enquêtes comparables Génération 1992, 1998, 2004, 2010, interrogations à 5 ans.

Avoir déjà travaillé permet d'échapper à cette situation, notamment s'il s'agissait d'un contrat à durée indéterminée, d'un emploi d'une durée d'au moins un an ou d'un contrat aidé. Cependant, à caractéristiques équivalentes, la population féminine a toujours plus de risques d'être NEET cinq ans après la sortie de formation. De plus, être une femme avec enfant influence aussi la probabilité de se déclarer en inactivité plutôt qu'au chômage, par comparaison à une femme sans enfant, tandis que pour les hommes (avec ou sans enfant), l'effet est non significatif.

Des distinctions apparaissent au vu des modèles séparés selon le genre. Au fil des générations, à caractéristiques équivalentes, les femmes deviennent moins souvent NEET évolution significative et contraire à celle des hommes - alors qu'elles affichent davantage leur priorité de ménager leur vie hors travail. Avoir eu le modèle d'une mère au foyer augmente aussi leur probabilité d'être NEET, ce qui n'est pas le cas chez les hommes. Une autre différenciation se trouve dans la vie de couple : le risque d'être NEET à cinq ans est plus élevé pour une femme en couple alors qu'il est plus faible pour un homme en couple. De plus, les femmes d'origine maghrébine sont davantage exposées aux risques de chômage et d'inactivité, cumulant les inégalités sociales, d'origine et de genre. 
Par ailleurs, les débuts de parcours influent sur le risque d'être NEET de manière accentuée du côté des femmes, surtout celles qui ont connu cette situation durant les six premiers mois de leur vie active et/ou trois ans après leur sortie. Les contrats à durée indéterminée ou de plus d'un an minorent les probabilités d'être NEET à cinq ans pour les deux populations, de même que les emplois aidés pour la population féminine seulement.

L'étude comparative des odds ratio dans le temps confirme aussi que si la maternité est un facteur déterminant pour les quatre cohortes, le risque diminue de Génération en Génération. De la sorte, les hommes (avec ou sans enfant) qui étaient moins exposés voient cet avantage se réduire au point d'observer, pour la dernière enquête, la même probabilité d'être NEET qu'une femme sans enfant. En contrepoint, le diplôme devient plus déterminant qu'il ne l'était en 1992. Il est ainsi aujourd'hui plus risqué d'être non diplômé que d'être une femme ${ }^{16}$. L'impact des autres caractéristiques individuelles (retard scolaire, profession et nationalité des parents) reste relativement constant.

Le profil de la population NEET a donc évolué sur vingt ans : alors qu'ils se définissaient par une féminisation élevée $(64 \%)$ pour la première génération, les NEET frôlent la parité pour les deux dernières Générations (47\%). Les niveaux de qualification plus élevés des femmes peuvent expliquer leur part moins importante parmi les NEET. Plus présentes sur le secteur des services générateur d'emplois (Milewski, 2010), et notamment celui « acyclique » des services publics (Gilles, 2012), elles tirent profit de leur réussite scolaire, le diplôme jouant un rôle protecteur contre les fluctuations conjoncturelles. En contraste, l'industrie et la construction, secteurs masculins durement touchés par les différentes perturbations économiques (1993,2001), et plus spécifiquement celle de 2008, tendent à réduire les offres d'embauche et à devenir parallèlement plus sensibles aux qualifications détenues, par le jeu de la concurrence entre demandeurs d'emploi. Ces contextes sectoriels sur un marché du travail segmenté ont entraîné un rapprochement des taux de chômage féminin et masculin (Coquet, op. cit. ; Danzin \& al., 2011). Les aléas économiques qui incitent les employeurs à proposer des formes d'emploi temporaire (contrats à durée déterminée, intérim, temps partiel) plutôt que des emplois stables, pourraient aussi jouer à l'avantage des femmes, peut-être plus intéressées par les possibilités qu'accordent les contrats temporaires de concilier vie de famille et vie professionnelle (Couprie, Joutard, 2017).

Toutefois, une analyse plus précise révèle que le changement dans la composition du public a essentiellement concerné les femmes avec enfant et les hommes sans enfant. En effet, pour les deux premières cohortes, femmes avec enfant, femmes sans enfant et hommes sans enfant représentaient respectivement environ un tiers de la population

16. Des modélisations logistiques ont été réalisées séparément pour chaque enquête, non présentées ici faute de place : une personne non diplômée de la génération 2010 a huit fois plus de risques de devenir NEET, alors que ce rapport n'était que de quatre en 1992 (cf. Danner \& al., 2018 p. 117). 
NEET à cinq ans. La catégorie des pères reste peu présente sur les quatre enquêtes (4\%), à la différence des hommes sans enfant qui composent désormais la moitié des NEET. Dans un mouvement inverse, les mères ne caractérisent plus que $18 \%$ des effectifs sur les deux dernières cohortes. Au vu de ce double mouvement qui a modifié la composition du public NEET en quatre Générations, l'idée selon laquelle les femmes sont susceptibles de rester au foyer lorsqu' elles ont des responsabilités familiales semble moins s'imposer lors deux dernières enquêtes.

En définitive, le poids des caractéristiques individuelles a évolué en vingt ans, à l'exception de l'origine sociale et de l'âge ${ }^{17}$. Parmi les NEET à cinq ans, se trouvent davantage de jeunes non diplômés, moins de femmes avec enfant, plus d'hommes sans enfant, des jeunes résidant plus souvent chez leurs parents. En effet, le maintien au domicile des parents concerne $56 \%$ de la cohorte 2010, au lieu de $41 \%$ pour les premières Générations. Si les situations des unes et des autres paraissent se rapprocher sur le marché du travail, les jeunes femmes n'en sortent pas gagnantes pour autant au regard de leurs trajectoires. Ainsi, la population féminine demeure moins nombreuse à connaître des parcours de transition linéaires de l'école à l'emploi. Or, le fait de passer par une situation donnée (NEET, emploi précaire...) marque-t-il durablement leur trajectoire individuelle tel un "effet cicatrice" (OCDE, 2010)?

\section{NEET un jour, NEET toujours?}

À travers les enquêtes du Céreq, vivre un temps de NEET est devenu un point de passage inévitable pour $74 \%$ des sortantes et $69 \%$ des sortants de chaque cohorte, sauf pour la première (80\% des femmes et $76 \%$ des hommes quittant l'école en 1992). Être NEET peut correspondre à un état transitoire, comme lors des premiers mois après la sortie de l'école, ou un moment plus ou moins persistant avec des allers et retours entre emplois temporaires ou temps de formation.

Dans les faits, très peu de jeunes restent NEET durant les cinq années suivant la sortie de l'école : $3 \%$ des femmes et $1 \%$ des hommes sur les quatre Générations. Ce faible pourcentage bouscule l'image quelque peu négative associée aux NEET comme étant celle de jeunes ne voulant ou ne pouvant pas travailler. Parmi les personnes retrouvées et répondant aux enquêtes, la représentation d'une jeunesse à la dérive et ne cherchant pas à s'insérer n'apparaît pas à travers les réponses. En revanche, l'étude des déterminants individuels permet de montrer que cette situation de « NEET toujours »

17. Quelle que soit la Génération, les jeunes NEET à 5 ans ont quitté le système éducatif à 20 ans, un an plus tôt que les autres jeunes (sans écart sexué), le redoublement en primaire constituant un jalon de leur cursus scolaire pour le tiers d'entre eux (versus $21 \%$ des autres jeunes en moyenne). 
touche plus souvent la population féminine non diplômée au fil des Générations, mais n'épargne pas la population masculine. Ainsi, la proportion de jeunes sans diplôme restés NEET durant les cinq années d'observation évolue de 7 à $15 \%$ chez les femmes, de 1 à $9 \%$ chez les hommes entre les Générations 1992 et 2010, alors que, parallèlement, le nombre de non-diplômés diminue.

Les caractéristiques individuelles déterminent non seulement la probabilité de connaître une situation NEET sur les cinq années après la fin des études, mais aussi la durée de cette situation. Quels que soient la Génération et le diplôme obtenu, les femmes cumulent toujours davantage de mois passés en marge de l'entreprise et de la formation (Tableau 4). Ainsi, les sortantes sans diplôme des trois premières cohortes ont expérimenté en moyenne 27 mois de NEET sur les cinq ans (17 mois pour les hommes), et lors de la dernière enquête, ce temps a augmenté jusqu'à 33 mois cumulés (30 mois pour les hommes).

Tableau 4. Temps moyen passé en NEET selon le diplôme (en mois)

\begin{tabular}{|c|c|c|c|c|c|c|c|c|}
\hline & \multicolumn{2}{|c|}{ Génération 92} & \multicolumn{2}{|c|}{ Génération 98} & \multicolumn{2}{|c|}{ Génération 2004} & \multicolumn{2}{|c|}{ Génération 2010} \\
\hline & Femmes & Hommes & Femmes & Hommes & Femmes & Hommes & Femmes & Hommes \\
\hline Non diplômé & 27 & 16 & 27 & 15 & 26 & 20 & 33 & 30 \\
\hline CAP-BEP & 19 & 9 & 18 & 8 & 18 & 11 & 23 & 17 \\
\hline Baccalauréat & 15 & 8 & 12 & 6 & 11 & 8 & 12 & 11 \\
\hline Supérieur & 11 & 8 & 8 & 5 & 8 & 7 & 9 & 7 \\
\hline Ensemble & 17 & 10 & 13 & 8 & 13 & 11 & 15 & 15 \\
\hline
\end{tabular}

Lecture : sur les cinq ans après leur sortie d'école, les femmes sorties sans diplôme de la Génération 2010 ont passé 33 mois en NEET - ni en étude, ni en emploi ni en formation, les femmes diplômées du supérieur 9 mois (en mois cumulés).

Sigle : CAP-BEP : respectivement certificat d'aptitude professionnelle et brevet d'études professionnelles.

Source : Céreq, enquêtes comparables Génération 1992, 1998, 2004, 2010, interrogations à 5 ans.

L'estimation en mois cumulés masque le fait que de nombreux jeunes alternent en réalité des expériences de travail et des épisodes sans emploi ni formation. En effet, les jeunes NEET observés à cinq ans ont eu un parcours professionnel avec de multiples emplois pour $33 \%$ des femmes et $41 \%$ des hommes en moyenne (Tableau 6). Quelle que soit la cohorte, les jeunes sont nombreux à avoir occupé un emploi d'au moins six mois (environ $70 \%$ des femmes et $76 \%$ des hommes), et plusieurs ont signé un contrat à durée indéterminée (près de $34 \%$ des femmes et $38 \%$ des hommes). Ces quelques chiffres permettent d'entrevoir les contours d'une segmentation sexuée du marché du travail, où les opportunités d'embauche en CDI et à temps complet sont toujours plus rares pour les femmes (Couprie, Joutard, op. cit.), d'autant que les tendances sur vingt ans soulignent une forte baisse de l'emploi à temps complet.

Autre constat, la première expérience de NEET dure en moyenne six mois chez les femmes (quatre mois chez les hommes) pour les trois premières Générations et sept mois pour la dernière Génération sans écart sexué. De plus, à l'issue de cette première séquence, les hommes se retrouvent plus souvent en emploi, alors que les femmes 
reprennent davantage des études ou une formation, une constante qui se retrouve sur les quatre enquêtes.

Pour connaître les déterminants quant à la probabilité de sortir de cette situation de NEET, un modèle de durée semi-paramétrique ${ }^{18}$ a été réalisé (Tableau 5). Le poids du milieu social est, de façon attendue, déterminante : le fait d'avoir ses deux parents en emploi, et notamment la mère, diminue la probabilité de rester NEET. En revanche, les jeunes d'origine maghrébine, femmes et hommes, sont davantage exposée.s aux risques de chômage et d'inactivité prolongés. Par ailleurs, la région où les jeunes ont fait leurs études, plus ou moins dynamique en termes de perspectives d'emploi ou de formation, influe aussi sur leur insertion. Le contexte local est aussi à prendre en considération dans la probabilité de sortir d'une situation de NEET (par exemple effet pénalisant dans le Nord-Pas-de-Calais où le taux de chômage demeure élevé, au contraire des régions Île-de-France et Rhône-Alpes). Autre résultat soulignant les dégradations conjoncturelles, la probabilité de sortie de cette situation s'est réduite au cours des enquêtes par rapport à la Génération 1992.

Tableau 5. Facteurs expliquant la sortie de la première séquence en NEET (modèle de durée de Cox)

\begin{tabular}{|l|c|c|c|}
\hline \multicolumn{1}{|c|}{ Variables } & Ensemble & Femmes & Hommes \\
\hline Femme Homme (Réf.) & $-0.294^{*}$ & & \\
Retard à l'entrée en 6 & $-0.0534^{*}$ & $-0.0654^{*}$ & $-0.0464^{*}$ \\
Sortant d'apprentissage & $0.0906^{*}$ & 0.0306 & $0.108^{* *}$ \\
Sans diplôme Sortie avec un CAP-BEP (Réf.) & $-0.351^{*}$ & $-0.317^{*}$ & $-0.358^{*}$ \\
Baccalauréat & $0.210^{*}$ & $0.252^{*}$ & $0.176^{*}$ \\
Diplôme Bac+2 ou 3 & $0.337^{*}$ & $0.432^{*}$ & $0.250^{*}$ \\
Diplôme Bac + 4 ou plus & $0.340^{*}$ & $0.433^{*}$ & $0.268^{*}$ \\
Formation générale Formation industrielle (Réf.) & $-0.231^{*}$ & $-0.138^{*}$ & $-0.250^{*}$ \\
Formation tertiaire (services) & $-0.0324^{*}$ & $0.112^{*}$ & $-0.0716^{*}$ \\
Arrêt des études pour raison financière & $0.0469^{*}$ & $0.104^{*}$ & 0.000964 \\
Arrêt des études par lassitude & $0.0357^{*}$ & $0.0487^{*}$ & 0.0234 \\
Parents indépendants Parents ouvriers employés (Réf.) & 0.0264 & $0.0550^{*}$ & -0.00310 \\
Parents cadres & 0.00984 & 0.0184 & 0.0144 \\
Parents techniciens & $0.0925^{*}$ & 0.0915 & 0.106 \\
Deux parents en emploi à la fin des études & $0.139^{*}$ & $0.121^{*}$ & $0.157^{*}$ \\
Mère au foyer à la fin des études & $-0.0945^{*}$ & $-0.136^{*}$ & $-0.0589^{*}$ \\
Parents Français Parents autres origines (Réf.) & $0.0998^{*}$ & $0.127^{*}$ & $0.0859^{*}$ \\
\hline
\end{tabular}

18. Le modèle de Cox (estimation semi-paramétrique) est mis en œuvre afin d'estimer les effets des variables socio-démographiques qui jouent sur la probabilité de sortie de la première période de NEET immédiatement consécutive à la fin des études. Ce modèle de régression permet d'étudier le temps écoulé avant qu'un événement ne survienne, ici l'obtention d'un emploi ou d'une formation, en fonction des différentes variables. 


\begin{tabular}{|c|c|c|c|}
\hline Parents d'Europe du sud & $0.142^{*}$ & $0.181^{*}$ & $0.0994^{*}$ \\
\hline Parents du Maghreb & $-0.115^{*}$ & $-0.124^{*}$ & $-0.123^{*}$ \\
\hline Ile-de-France Fin des études autres régions (Réf.) & $0.0519^{*}$ & $0.137^{*}$ & -0.0186 \\
\hline Nord-Pas-de-Calais & $-0.138^{*}$ & $-0.222^{*}$ & $-0.0835^{*}$ \\
\hline Alsace & $0.208^{*}$ & $0.211^{*}$ & $0.208^{*}$ \\
\hline Bretagne & $0.0652^{*}$ & $0.0775^{*}$ & 0.0604 \\
\hline Pays-de-Loire & $0.161^{*}$ & $0.172^{*}$ & $0.146^{*}$ \\
\hline Aquitaine & 0.0133 & 0.0669 & -0.0363 \\
\hline Midi-Pyrénées & -0.00423 & 0.0148 & -0.0251 \\
\hline Rhône-Alpes & $0.0974^{*}$ & $0.114^{*}$ & $0.0729^{*}$ \\
\hline PACA (Provence-Alpes-Côte d'Azur) & $-0.0490^{*}$ & -0.0523 & -0.0519 \\
\hline Avoir connu une expérience professionnelle avant neet & $0.246^{*}$ & $0.284^{*}$ & $0.196^{*}$ \\
\hline Avoir connu une expérience professionnelle avant neet (carré) & $-0.000240^{*}$ & $-0.000302^{*}$ & $-0.000194^{*}$ \\
\hline Génération 1992 (Réf.) & $-0.0351^{*}$ & 0.0355 & $-0.0975^{*}$ \\
\hline Génération 2004 & $-0.0837^{*}$ & $0.0889^{*}$ & $-0.224^{*}$ \\
\hline Génération 2010 & $-0.295^{*}$ & $-0.0572^{*}$ & $-0.481^{*}$ \\
\hline N & 51807 & 24422 & 27385 \\
\hline Log de vraissemblance & -453258.0 & -191312.1 & -230691.0 \\
\hline
\end{tabular}

Sont indiqués les résultats aux seuils significatifs de $5 \%$.

Lecture : toutes choses égales par ailleurs, une femme a moins de chances de sortir d'une première expérience de NEET (ni en étude, ni en emploi, ni en formation) vers l'emploi ou la formation qu'un homme (coefficient négatif de 0,294 significatif au seuil de $5 \%$ ).

Sigle : CAP-BEP : respectivement certificat d'aptitude professionnelle et brevet d'études professionnelles.

Source : Céreq, enquêtes comparables Génération 1992, 1998, 2004, 2010, interrogations à 5 ans.

Toutes choses égales par ailleurs (ou à caractéristiques équivalentes), la transition vers l'emploi ou la formation augmente avec l'élévation de la qualification et concerne davantage les jeunes sans retard scolaire, les femmes formées plutôt dans une spécialité tertiaire, les hommes dans une spécialité industrielle, voire pour ces derniers, ayant terminé par un contrat d'apprentissage. La force du diplôme ressort davantage pour la population féminine. Toutefois, à caractéristiques équivalentes, une femme a toujours moins de chances de sortir de cette situation par rapport à un homme (- $25 \%)$. L'expérience sur le marché du travail (nombre de mois en emploi) avant le premier passage en NEET a aussi un fort impact positif (+ $27 \%)$, de manière accentuée chez les femmes. Le passage de l'école à l'emploi reste donc une période critique dans la vie des jeunes parce que leurs premiers pas sur le marché du travail peuvent avoir un effet significatif sur leur trajectoire.

L'insertion professionnelle de ces jeunes, rythmée par des alternances entre emplois précaires et expériences de NEET, tel un flux et reflux sur le marché du travail, entraîne aussi une "désynchronisation" des étapes de leur transition vers l'âge adulte (Galland, 2011). Si les femmes quittent le domicile parental toujours plus tôt que les hommes 
et vivent plus souvent en couple, cette tendance a significativement diminué ${ }^{19}$. En revanche, ce mode de vie reste une modalité dominante pour les hommes : plus des trois quarts des hommes NEET à cinq ans demeurent chez leurs parents, quelle que soit la cohorte, la mise en couple restant faible ${ }^{20}$. L'accès à une autonomie résidentielle s'est affaibli et le maintien au foyer parental peut se lire comme le résultat d'un choix ou d'une contrainte liée au manque de ressources financières.

En fait, le terme NEET masque ainsi un éventail de profils : jeunes hors de l'emploi et de la formation de courte ou longue durée, avec des responsabilités parentales ou non, en couple ou résidant chez leurs parents, diplômé.e.s ou peu... Il masque aussi des réalités différenciées chez les hommes et les femmes, mises en lumière au regard des enquêtes du Céreq.

\section{Pour un autre regard sur les mondes NEET}

L'indicateur NEET n'est pas un concept qui a émergé d'une réflexion scientifique adossée à un champ théorique. Il est devenu une catégorie cible des politiques publiques, adoptée par un ensemble de pays soucieux de se saisir des multiples problèmes rencontrés par les jeunes, dont celui de ne pas accumuler de capital humain, pouvant entraîner une exclusion du marché du travail, voire sociale, susceptible de conséquences préjudiciables pour l'individu, la société et l'économie (non-protection sociale, trappe à pauvreté...). Si cette non-participation au marché du travail des jeunes est un problème ancien, la nouveauté a été sa progression liée à la crise financière et économique de 2008 (Carcillo \& al., op. cit.). De plus, contrairement à d'autres pays de l'Union européenne, la part de NEET n'a pas baissé depuis 2013, en France, ce qui souligne la pertinence de mieux appréhender cette population.

Cet indicateur s'avère donc utile pour apprécier la part croissante des jeunes qui échappent aux espaces habituels d'intégration sociale (école, emploi) et pour mesurer les conséquences sociales sur leurs trajectoires de ces épisodes où ils restent plus ou moins longtemps à l'écart de la société. Au vu des frontières relativement floues entre chômage et inactivité (cf. Le halo du chômage, Bessone \& al., op. cit.), il permet de franchir les ornières liées à la dimension restrictive (et déformante) d'une réflexion qui ne porte que sur les actifs, et de raisonner sur l'ensemble des jeunes, y compris ceux en étude ou formation. Ainsi, en brisant la barrière chômage/inactivité, il s'agit de rendre visible une population

19. Parmi les femmes NEET à cinq ans, la vie en couple concerne $46 \%$ de la cohorte 2010 au lieu de $60 \%$ pour les premières Générations (Tableau 6).

20. Diminuant de 21 à $11 \%$ des hommes sortis en 1992 et 2010. 
par rapport à l'ensemble de la jeunesse et de questionner son désengagement ou son exclusion du marché du travail, voire son exclusion sociale.

Cette recherche revient toutefois sur l'importance de dépasser l'utilité statistique d'un tel indicateur statique rassemblant, sous une même identité, des jeunes de profils variés pour considérer les réalités humaines qu'il recouvre. Or, de ce point de vue, il peut aussi bien refléter une situation contrainte ${ }^{21}$ ou acceptée, voire choisie. Certains jeunes NEET paraissent ainsi cumuler des caractéristiques qui font peser sur eux un risque de marginalisation et d'exclusion. Le regard se tourne en particulier vers les sortants sans diplôme (40\% des NEET) qui rencontrent des difficultés à s'insérer professionnellement, d'autant plus conséquentes que le contexte économique est défavorable.

D’autres jeunes, identifiés aussi comme NEET, refusent des emplois précaires en décalage avec leurs qualifications et leurs attentes, ou reportent leur projet d'insertion professionnelle pour assumer une responsabilité parentale ou encore retournent à leur avantage une situation sociale imposée (Schehr, 1999) pour investir d'autres valeurs que celles du travail et de la formation (famille, année sabbatique, loisir, créativité artistique...). À titre d'exemple, sur toutes les enquêtes, plus de $40 \%$ des femmes avec enfant affirment que la situation actuelle leur convient ${ }^{22}$ (versus moins du quart des autres NEET hommes ou femmes), et leur priorité est de ménager leur vie hors travail pour plus du quart d'entre elles $^{23}$. Il y aurait cependant matière à s'interroger sur le vécu et la réalité de la vie des femmes derrière ces réponses, comme invitait à le faire Margaret Maruani à propos du travail à temps partiel (2004, p. 111) : que signifie être NEET « quand les pressions sont tellement fortes qu'il n'y a pas d'autres solutions? Quand les emplois proposés ne sont jamais à temps plein? Quand les contraintes de la vie familiale deviennent trop complexes?". La question n'est pas tant de savoir si être NEET convient « un peu, beaucoup ou pas du tout, mais d'en voir les conséquences".

De plus, les résultats interpellent les représentations sur les NEET, souvent associées au désengagement des jeunes vis-à-vis du marché du travail, du monde économique et plus largement de la société. Être NEET est connoté dans le débat social, et définit le jeune par la négative, par ce qu'il ne fait pas et/ou n'est pas (ni en emploi, ni en formation, ni en étude), quitte à l'enfermer dans la case statique d'une « jeunesse à la dérive » qui n'apparaît cependant pas à travers les enquêtes du Céreq. Le travail garde une importance certaine pour ces jeunes (Sarfati, 2015).

21. Élargissant la théorie du capital humain, l'approche par les capacités de Sen (2005) s’intéresse aux ressources et aux opportunités dont dispose un individu : ceux dont la situation scolaire et sociale parait la moins favorable ont de fait moins de liberté d'action, moins de possibilités de se définir dans la société et subissent donc davantage les situations.

22. La question posée par le Céreq pour les quatre enquêtes était ainsi formulée : "Aujourd’hui, vous diriez que la situation actuelle vous convient ou ne vous convient pas?", avec des nuances de formulation (par exemple professionnelle) qui mériteraient débat sur l'effet quant à la qualité des réponses.

23. Versus moins de $10 \%$ des autres NEET et $17 \%$ des jeunes en emploi et formation. 
Tableau 6. Portraits des jeunes NEET cinq ans après leur sortie de formation (en \%)

\begin{tabular}{|c|c|c|c|c|c|c|c|c|}
\hline & \multicolumn{2}{|c|}{$\begin{array}{c}\text { Génération } \\
1992\end{array}$} & \multicolumn{2}{|c|}{$\begin{array}{c}\text { Génération } \\
1998\end{array}$} & \multicolumn{2}{|c|}{$\begin{array}{l}\text { Génération } \\
2004\end{array}$} & \multicolumn{2}{|c|}{$\begin{array}{c}\text { Génération } \\
2010\end{array}$} \\
\hline & Femmes & Hommes & Femmes & Hommes & Femmes & Hommes & Femmes & Hommes \\
\hline $\begin{array}{l}\text { Âge moyen à la } \\
\text { sortie }\end{array}$ & 20 ans & 20 ans & 20 ans & 20 ans & 20 ans & 20 ans & 20 ans & 19 ans \\
\hline Vivent en couple & 64 & 21 & 60 & 17 & 52 & 16 & 46 & 11 \\
\hline $\begin{array}{l}\text { Vivent au } \\
\text { domicile parental }\end{array}$ & 26 & 71 & 25 & 64 & 31 & 64 & 37 & 72 \\
\hline $\begin{array}{l}\text { Ont un enfant } \\
\text { et plus }\end{array}$ & 52 & 9 & 53 & 10 & 42 & 5 & 40 & 7 \\
\hline $\begin{array}{l}\text { Ont eu: } \\
\text { - plusieurs } \\
\text { emplois }\end{array}$ & 37 & 43 & 32 & 43 & 37 & 47 & 28 & 32 \\
\hline $\begin{array}{l}\text { - un emploi de } \\
\text { plus de } 6 \text { mois }\end{array}$ & 77 & 78 & 73 & 82 & 70 & 80 & 61 & 63 \\
\hline - un CDI & 35 & 35 & 36 & 44 & 34 & 38 & 32 & 34 \\
\hline $\begin{array}{l}\text { Situation actuelle } \\
\text { convient }\end{array}$ & 29 & 15 & 37 & 21 & 28 & 20 & 33 & 23 \\
\hline $\begin{array}{l}\text { Ménager sa vie } \\
\text { hors travail }\end{array}$ & 25 & 14 & 26 & 18 & 15 & 8 & 16 & 7 \\
\hline $\begin{array}{l}\text { Trouver un } \\
\text { emploi stable }\end{array}$ & - & - & 62 & 62 & 67 & 69 & 65 & 71 \\
\hline $\begin{array}{l}\text { Inquiet sur } \\
\text { l'avenir } \\
\text { professionnel }\end{array}$ & 65 & 58 & 39 & 36 & - & - & 44 & 37 \\
\hline $\begin{array}{l}\text { Recherchent un } \\
\text { emploi }\end{array}$ & 86 & 97 & 76 & 88 & 72 & 86 & 74 & 86 \\
\hline $\begin{array}{l}\text { Ont des } \\
\text { indemnités, } \\
\text { allocations }\end{array}$ & 67 & 61 & 82 & 61 & 72 & 56 & 69 & 48 \\
\hline
\end{tabular}

Lecture : pour la Génération 2010, parmi les femmes NEET (ni en étude, ni en emploi, ni en formation), $46 \%$ vivent en couple, 37 \% vivent au domicile de leurs parents, $40 \%$ ont un ou plusieurs enfants... cinq ans après la fin des études.

Sigles : CDI : contrat à durée indéterminée.

Source : Céreq, enquêtes comparables Génération 1992, 1998, 2004, 2010, interrogations à 5 ans.

La plupart ont déjà connu des emplois, voire un contrat à durée indéterminée et leur priorité majeure, affirmée par environ $64 \%$ des femmes et $68 \%$ des hommes NEET au moment des enquêtes, est bien de (re)trouver un emploi stable (Tableau 6). De Génération en Génération, elles et ils effectuent de nombreuses démarches de recherche d'emploi (candidatures spontanées, réponses à des annonces, inscription dans une agence d'intérim...) et s'inscrivent à Pôle Emploi (Agence nationale pour l'emploi) ou à l'Apec (Association pour l'emploi des cadres) : $77 \%$ des femmes et $88 \%$ des hommes en moyenne sur les quatre enquêtes. Certain·ess envisagent même de se mettre à leur 
compte avec toujours une différenciation sexuée, les femmes exprimant moins ce projet ${ }^{24}$. De façon globale, ces jeunes sont plus nombreux à exprimer de l'inquiétude quant à leur avenir professionnel, de manière accentuée chez la population féminine (44\% des femmes et $37 \%$ des hommes sortant en 2010 versus $19 \%$ des personnes non NEET). Les femmes se montrent toujours plus inquiètes, quelle que soit la Génération, et ce sentiment d'insécurité est pour partie lié à leur position vis-à-vis de l'emploi, aux épreuves traversées (études inachevées, mauvaise santé, sentiment de discrimination... (cf. Béduwé, Dupray, 2018). Être NEET cinq ans après la sortie de l'école est une situation qui ne leur convient pas pour plus des deux tiers, même si ces taux fluctuent légèrement selon les enquêtes.

Ce portrait des NEET invite donc à s'interroger sur les raisons qui ont conduit cette jeunesse à se trouver dans cette situation à un moment donné de leur trajectoire. Comment les jeunes s'approprient-ils les épisodes d'allers-retours vers la situation de NEET ? Appréhender cette question suppose aujourd'hui de développer des recherches en s'intéressant davantage à leur vécu, leurs expériences, leur rapport au travail, les obstacles rencontrés, leurs opportunités, les moments de ruptures de parcours... Il s'agit aussi d'étudier les attentes, les logiques ou stratégies des personnes qui restent déconnectées des marchés de l'emploi et de la formation.

\section{Conclusion}

Ainsi, il n'existe pas un modèle unique d'accès à la situation de NEET : connaître une expérience NEET dans son parcours peut aussi bien se présenter comme une alternative personnelle, quand d'autres jeunes sont dans des configurations qui les rendent vulnérables par rapport à l'insertion (faible qualification, problèmes de santé, de discrimination...). Toutefois, les résultats de cette recherche rappellent qu'un épisode NEET dès la sortie du système éducatif a des répercussions sur les trajectoires.

Or, ces constats ne peuvent faire l'économie d'une analyse genrée dans la mesure où les femmes présentent des caractéristiques (plus diplômées ${ }^{25}$, plus souvent polarisées sur les formations en lien avec les services, davantage préoccupées par l'articulation entre vies professionnelle et familiale...) qui ne les positionnent pas de manière comparable aux hommes par rapport aux enjeux d'insertion sociale et professionnelle. Ces expériences ne se déclinent pas de la même manière au féminin et au masculin : connaître une séquence NEET enferme davantage les femmes, notamment les moins diplômées, dans cette situation.

24. Près de $30 \%$ des femmes et la moitié des hommes exprimaient ce projet au cours des deux premières enquêtes, pour $17 \%$ des femmes et $27 \%$ des hommes sortant en 2010 .

25. Ainsi, en moyenne, sur les quatre enquêtes, $37 \%$ des femmes et $32 \%$ des hommes ont quitté l'école avec un diplôme de l'enseignement supérieur, et $16 \%$ des femmes et $23 \%$ des hommes sont sortis sans aucun diplôme.. 
Au-delà du genre, d'autres facteurs sociaux, familiaux, contextuels tendent à expliquer cette déconnexion de l'emploi et de la formation, mais l'analyse confirme que le capital éducatif reste le premier déterminant du risque de devenir NEET, qui se renforce au fil des ans. Cette recherche dynamique et comparative sur quatre enquêtes a permis d'apprécier le poids de ces différents facteurs pouvant expliquer comment les jeunes deviennent et restent NEET, en France.

Cependant, la situation NEET peut aussi être le résultat ou les conséquences de problèmes structurels liés au marché du travail (segmentation, qualité des emplois, temps partiels...), aux politiques d'emploi et de formation et aux caractéristiques des politiques sociales du pays où vivent les jeunes (Cuzzocrea, 2014), comme les allocations chômage accordées aux personnes qui ont travaillé un certain laps de temps. Près des trois quarts des femmes NEET au moment des enquêtes perçoivent des indemnités de chômage et des allocations familiales et financières régulières en fonction de leurs responsabilités familiales (RMI - revenu minimum d'insertion -, aide parent isolé, aide au logement). Ces indemnités sont aussi perçues par plus de la moitié des hommes NEET (Tableau 5).

Nos résultats confortent l'intérêt de poursuivre l'analyse genrée en faisant l'hypothèse que les problématiques des hommes et des femmes n'attendent pas une réponse uniforme en termes d'actions sociales ou de politiques publiques (soutien pour la garde d'enfants, la mobilité, la reprise d'études...).

La catégorie de NEET amalgame par conséquent des populations et des situations assez hétérogènes entre les mots et les maux qu'elle est supposée décrire. Finalement, quelle est la plus-value de cet indicateur par rapport à une analyse partielle sur le chômage ou l'inactivité ? Sur les déterminants du non-emploi, aucune. En revanche, le fait de proposer un seul indicateur pour saisir les situations de chômage et d'inactivité permet d'intégrer les femmes, souvent invisibles dans les statistiques sur l'insertion. Comme le soulignait Margaret Maruani en 2002 (p. 58), "Linactivité, dans bien des cas, masque le chômage et doit être étudiée en tant que telle: une des formes d'exclusion du marché du travail, une des façons de gommer le chômage ". Le concept de NEET offre en fait l'avantage (ou le désavantage) de prendre en compte la diversité des situations des femmes et des hommes et de dévoiler des effets genrés dans l'étude des trajectoires sur le marché du travail (GuergoatLarivière, Lemière, $o p$. cit.).

\section{Bibliographie}

Barret C., Ryk F., Volle N. (2014), «Enquête 2013 auprès de la Génération 2010. Face à la crise, le fossé se creuse entre niveaux de diplôme ", Céreq-Bref, n 319.

Béduwé C., Dupray A. (2018) «D’une Génération à l'autre : l'inquiétude des jeunes en question ", Céreq-Bref, n 361. 
Bessone A.-J., Cabannes P.-Y., Marrakchi A. (2016), "Halo autour du chômage : une population hétérogène et une situation transitoire ", Insee Références, pp. 21-33.

Bourdieu P., Passeron J.-C. (1970), La reproduction. Elément pour une théorie du système d'enseignement, Paris, Minuit.

Brinbaum Y., Guégnard C. (2012), « Parcours de formation et d'insertion des jeunes issus de l'immigration au prisme de l'orientation ", Formation Emploi, n 118, pp. 61-82.

Buisson G., Daguet F. (2012), "Qui vit seul dans son logement ? Qui vit en couple ? ", Insee Première, $\mathrm{n}^{\circ} 1392$.

Carcillo S., Fernández R., Königs S., Minea A. (2015), "NEET Youth in the Aftermath of the Crisis: Challenges and Policies", OECD Social, Employment and Migration Working Papers, 164, OECD.

Caroli E., Gautié J. (2009), Bas salaire et qualité de l'emploi : l'exception française ?, Paris, Édition Rue d'Ulm.

Coleman J. S. (1988), "Social capital in the creation of human capital", American journal of sociology, S95-S120.

Coquet B. (2004), "Les femmes face au chômage : une inégalité en déclin ", Revue de l'OFCE, $\mathrm{n}^{\circ}$ 90, pp. 117-151.

Couppié T., Épiphane D. (2018), "La relation genre-insertion a-t-elle évolué en 20 ans?", Essentiels, n 1, Céreq, pp. 141-149.

Couppié T., Épiphane D. (2007), «Vivre en couple et être parent : impacts sur les débuts de carrière ", Céreq-Bref, n 241.

Couprie H., Joutard X. (2017), « La place des emplois atypiques dans les trajectoires d'entrée dans la vie active ", Revue française d'économie, Vol. XXXII, pp. 59-93.

Cusset P.-Y., Garner H., Harfi M., Lainé F., Marguerit D. (2015), "Jeunes issus de l’immigration : quels obstacles à leur insertion économique? ", La note d'analyse, France Stratégie, Hors-série.

Cuzzocrea V. (2014), "La catégorie des Neet : quel avenir ? ", in Conseil de l'Europe, Points de vue sur la jeunesse, 2020, quelles perspectives ?, pp. 73-87.

Daguet F., Niel X. (2010), «Vivre en couple. La proportion de jeunes en couple se stabilise ", Insee Première, n ${ }^{\circ} 1281$.

Danner M., Guégnard C., Joseph O. (2018), «Alice au pays des NEET : la traversée du miroir sur 20 ans ", in Boudesseul G. \& al., Céreq Échanges, n 6, pp. 113-126.

Danzin E., Simonnet V., Trancart D. (2011), «L'impact de la crise sur les trajectoires professionnelles des jeunes ", Document de travail, $\mathrm{n}^{\circ}$ 146, Centre d'études pour l'emploi. 
Defresne F., Krop J. (2016), «La massification scolaire sous la Ve République. Une mise en perspective des statistiques de l'Éducation nationale (1958-2014) ", Education \& Formations, $\mathrm{n}^{\circ}$ 91, MENESR-DEPP.

Escudero V., Mourelo E. L. (2018), « La Garantie européenne pour la jeunesse. Bilan systématique des mises en œuvre dans les pays membres ", Travail et Emploi, n 153 , pp. 89-118.

European Commission (2012), Towards a Job-Rich Recovery, 173 final, Brussels, European commission.

European Commission (2010), Youth on the Move, Luxembourg, publications Office of the European Union.

European Council (2013), Council recommendation of 22 April on Establishing a Youth Guarantee, Brussels, European council.

Eurofound (2016), Exploring the diversity of NEET, Luxembourg, Publications Office of the European Union.

Eurofound (2012), NEETs. Young people not in employment, education or training: Characteristics, costs and policy responses in Europe, Luxembourg, Publications Office of the European Union.

Fondeur Y., Minni C. (2004), «L'emploi des jeunes au cœur des dynamiques du marché du travail ", Économie et Statistiques, Insee, n⿳ 374-379, pp. 85-104.

France Stratégie (2017), L’insertion professionnelle des jeunes, rapport de diagnostic élaboré par France stratégie et la Dares.

Freyssinet J. (2009), "La mesure du chômage : un enjeu de société ", Emploi et chômage dans les pays de l'OCDE : les grandes tendances, in Montel-Dumont O., Travail, emploi, chômage, Cahiers français, ${ }^{\circ}$ 353, pp. 10-15.

Furlong A. (2006), "Not a very NEET solution", Work, Employment and Society, 20, pp. 553-559.

Galland O. (2011), Sociologie de la jeunesse, Paris, Armand Colin.

Gautié J. (2009), Le chômage, Paris, La Découverte, collection « Repères ».

Gilles C. (2012), «L'emploi des femmes et des hommes dans la crise ", La note d'analyse, France Stratégie, ${ }^{\circ} 312$.

Granovetter M.S. (1995), Getting a Job. A Study of Contacts and Careers, The University of Chicago Press [ $1^{\text {re }}$ édition 1974].

Guégnard C., Joseph O. Murdoch J. (2015), « Au-delà de la crise en France, la vulnérabilité des jeunes d'origine maghrébine ", in Abecassis P., Coutinet N. (dir.), Économie 
sociale : crises et renouveaux, Cahiers du CIRTES Hors-Série, UCL Presses universitaires de Louvain, pp. 525-544.

Guergoat-Larivière M., Lemière S. (2014), « Emploi, non-emploi : une analyse femmeshommes ", Document de travail, $\mathrm{n}^{\circ} 176$, CEE.

Istance D., Rees G., Williamson H. (1994), Young people not in education, training or employment in South Glamorgan, Cardiff, UK, South Glamorgan Training and Enterprise Council.

Joseph O., Lopez A., Ryck F. (2008), "Génération 2004, des jeunes pénalisés par la conjoncture ", Céreq-Bref, n 248.

Kramartz F., Viarengo M. (2015), Ni en emploi, ni en formation, des jeunes laissés pour compte, Presses de Sciences Po.

De Larquier G., Rieucau G. (2012), « Comment êtes-vous entré(e) dans votre entreprise ? Les enseignements des enquêtes Emploi 2003-2011 ", Document de travail, CEE, $n^{\circ} 158$.

Martinelli D., Minni C. (2013), «Face à la crise, le diplôme protège du chômage et favorise l'accès à la formation continue ", Formations et Emploi, Insee.

Maruani M. (2004), "Activité, précarité, chômage : toujours plus ? ", Revue de l'OFCE 2004/3, n 90 , pp. 95-115.

Maruani M. (2002), Les mécomptes du chômage, Éditions Bayard.

Milewski F. (2010), "Chômage et emploi des femmes dans la crise en France ", Lettre de l'OFCE, $\mathrm{n}^{\circ} 31$, pp. 1-8.

OCDE (2010), Off to a good start? Jobs for youth, Paris, OECD.

OCDE (2009), Jobs for Youth, France, Paris, OECD.

Piore M.J. (1978), "Dualism in the labor market: A response to uncertainty and flux. The case of France", Revue économique, pp. 26-48.

Portes A. (2000), "Social capital: Its origins and applications in modern sociology", Annual review of Sociology, Vol. 24, pp. 1-24.

Quintini G., Martin S. (2006), Starting well or losing their way? The position of youth in the labour market in the OECD countries, Paris, OECD.

Rose J. (2018), "L'insertion professionnelle : une notion discutée mais robuste ", in Couppié \& al., 20 ans d'insertion professionnelle : entre permanences et évolutions, Essentiels, n 1 , Céreq, pp. 15-19.

Rose J. (2005), « D’une génération à l'autre, les effets de la formation initiale sur l'insertion ", Céreq-Bref, n 122. 
Ryan P. (2001), “The school-to-work transition: A cross-national perspective”, Journal of Economic Literature, Vol. 39, pp. 34-92.

Salais R., Baverez N., Reynaud B. (1986), L'invention du chômage, Paris, PUF.

Sarfati F. (2015), "L'insertion professionnelle des jeunes entre précarité, incertitude et expérimentation ", Cahiers de l'action, n 45, INJEP, pp. 9-16.

Schehr S. (1999), La vie quotidienne des jeunes chômeurs, Paris, PUF.

Sen A. (2005), Rationalité et liberté en économie, Paris, Odile Jacob.

Social Exclusion Unit (1999), Bridging the Gap: New Opportunities for 16-18 Year-Olds Not in Education, Employment or Training, London, Social Exclusion Unit, CM4405.

Williamson H. (1997), "Status Zer0, youth and the "underclass": Some considerations", in Youth, the "underclass" and social exclusion Routledge, London, UK, Mac Donald R. 